\title{
Estudo psicométrico das escalas CARSAL e CARVAL numa amostra de adolescentes em contexto insular
}

\section{Psychometric study of the CARSAL and CARVAL scales in a sample of adolescents in an insular context}

\author{
(D) José Mendes ${ }^{1} \bowtie$ \\ D Márcio Tavares ${ }^{2}$ \\ (iD) Teresa Medeiros ${ }^{3}$
}

\author{
[1] INTELECTO - Psicologia \& Investigação, Portugal \\ [2] Escola Superior de Saúde de Universidade dos Açores, Portugal \\ [3] Faculdade de Ciências Sociais e Humanas de Universidade dos Açores, Portugal \\ $\checkmark$ josemendes@intelecto.pt
}

\begin{abstract}
Resumo
Objetivo: A imagem corporal é muito relevante no período de desenvolvimento da adolescência. O presente estudo pretende analisar as propriedades psicométricos das escalas CARSAL e CARVAL (medidas da saliência e valência) numa amostra de adolescentes portugueses, residentes em contexto insular. Método: Estudo quantitativo, psicométrico e exploratório, seguiu os procedimentos éticos inerentes à amostra. Participaram 196 adolescentes, eliminando-se 4 participantes por não cumprirem os critérios de inclusão. Elaborou-se um questionário sociodemográfico e aplicaram-se as escalas CARSAL e CARVAL e a versão reduzida (30 itens) da Escala de Avaliação do Autoconceito de Adolescentes Piers-Harris. Resultados: Os adolescentes apresentaram idades compreendidas entre os 10 e os 17 anos, frequentam os segundo e terceiro ciclos do ensino básico e secundário. As escalas CARSAL e CARVAL apresentaram um bom índice de consistência interna, valores idênticos à versão original e versão adaptada e validada numa amostra de adultos portugueses. O modelo de análise fatorial confirmatória para a presente amostra de adolescentes, apresentou um ajustamento sofrível que após refinação do modelo pelas maiores covariâncias dos itens $7,8,9,10,11$ e $13\left(X^{2} / \mathrm{df}=3.377\right.$; GFI $=.88$; CFI $=.91 ; \mathrm{TLI}=.87 ; \mathrm{RMSEA}=.11 ; p[$ rmsea $\leq .05]=<.001)$, evidencia um melhor ajustamento, mas não aceitável. Analisou-se a escala CARVAL que apresenta fiabilidade e validade de constructo considerada adequada e um modelo considerado aceitável $\left(X^{2} / \mathrm{df}=2.59 ; \mathrm{CFI}=.98 ; \mathrm{GFI}=.95 ; \mathrm{TLI}=.96 ; \mathrm{RMSEA}=.09 ; \mathrm{p}[\mathrm{rmsea} \leq .05]=\right.$ .017). Conclusões: A escala CARSAL (saliência) não se revelou psicometricamente robusta para esta amostra de participantes adolescentes. Por outro lado, a escala CARVAL (valência) apresenta-se uma escala de oito itens psicometricamente robusta na avaliação do auto-esquema da aparência (avaliação positiva ou negativa da aparência) nesta amostra de adolescentes, residente na Região Autónoma dos Açores. Sugerem-se estudos futuros para uma amostra de adolescentes em outras regiões de Portugal.
\end{abstract}

Palavras-chave: Esquemas da aparência; Adolescentes; Autoconceito; Saliência; Valência.

\begin{abstract}
Objective: Body image is very relevant in the period of adolescence development. This study aims to analyze the psychometric properties of the CARSAL and CARVAL scales (measures of salience and valence) in a sample of Portuguese adolescents living in an insular context. Method: Quantitative, psychometric, and exploratory studies followed the ethical procedures inherent to the sample. A total of 200 adolescents participated, but 4 participants were eliminated for not meeting the inclusion criteria. A sociodemographic questionnaire was developed, the CARSAL and CARVAL scales and the short version of the Piers-Harris Adolescent Self-Concept Assessment Scale (30 items) were applied. Results: Adolescents are aged between 10 and 17 years old, attend the second and third cycles of basic and secondary education. The CARSAL and CARVAL scales showed a good index of internal consistency. Values identical to the original version and the adapted and validated version in a sample of Portuguese adults. The confirmatory factor analysis model for the present sample of adolescents showed a poor adjustment that after refining the model by the greater covariances of items $7,8,9,10,11$ and $13\left(X^{2} / \mathrm{df}=3.377\right.$; $\mathrm{GFI}=.88 ; \mathrm{CFI}=.91 ; \mathrm{TLI}=.87 ; \mathrm{RMSEA}=.11 ; p[\mathrm{rmsea} \leq .05]=<.001)$, shows a better fit, but not acceptable. The CARVAL scale was analyzed, which presents reliability and construct validity considered adequate and a model considered acceptable $\left(X^{2} / \mathrm{df}=2.59 ; \mathrm{CFI}=.98 ; \mathrm{GFI}=.95 ; \mathrm{TLI}=.96 ; \mathrm{RMSEA}=.09 ; p[\mathrm{rmsea} \leq .05]=\right.$ .017). Conclusions: The CARSAL (salience) scale was not psychometrically robust for this sample of adolescent participants. On the other hand, the CARVAL (valence) scale is a psychometrically robust eight-item scale in the assessment of self-scheme of appearance (positive or negative assessment of appearance) in this sample of adolescents living in the Autonomous Region of the Azores. Future studies are suggested for a sample of adolescents in other regions of Portugal.
\end{abstract}

Keywords: Appearance schemes; Adolescents; Self-concept; Boss; Valence

https://doi.org/10.52014/rppa.v1.i1.2021.20 


\section{INTRODUÇÃO}

A imagem do corpo ou imagem corporal, consiste numa relação pessoal com o próprio corpo, em que estão presentes variáveis como pensamentos, crenças, sentimentos e atitudes que dizem respeito à aparência física do indivíduo (Cash, 2008), é um processo de construção e transformação ao longo do ciclo vital.

No desenvolvimento da adolescência, a imagem corporal é uma variável psicológica e interpessoal muito importante (Ricciardelli \& Yager, 2016) e, provavelmente, a componente mais relevante para autoestima global dos adolescentes (Levine \& Smolak, 2004) e uma componente vital do autoconceito, referindo-se a conceções e atitudes que os indivíduos têm em relação ao seu próprio corpo (Hockenberry et al., 2013).

Aliadas ao desenvolvimento dos adolescentes estão as mudanças físicas na puberdade que ocorrem da atividade hormonal (Wong et al., 2011), em que por volta dos 10 anos de idade se dá um aumento rápido na altura e mudanças no peso, músculos e ossos, sendo esse desenvolvimento mais precoce nas raparigas (10-14 anos), quando comparados com rapazes, em que essas alterações ocorrem entre 11-16 anos (Papalia \& Feldman, 2013). Assim, as preocupações com a imagem corporal são comuns na adolescência (Ricciardelli \& Yager, 2016), considerada como um período crucial do desenvolvimento (Medeiros, 2013). Segundo a Organização Mundial da Saúde (OMS), a adolescência compreende o intervalo entre os 10-19 anos, período de tempo em que o indivíduo se transforma, com o aparecimento de características sexuais secundárias (puberdade), até atingir a maturidade sexual e reprodutiva (Hockenberry et al., 2013; Papalia \& Feldman, 2013). Na puberdade, com o aparecimento dos caracteres sexuais secundários (e.g., pelos pubianos e axilares, aparecimento das mamas, aumento da largura e profundidade da pélvis, mudanças na pele e na voz, na rapariga; pelos pubianos, axilares e faciais, alargamento dos ombros, bem como mudanças na pele e na voz, no rapaz) e o desenvolvimento dos órgãos reprodutores, alterase a imagem corporal. Grogan (2017) defende que as alterações físicas e psicológicas que ocorrem neste período de desenvolvimento humano influenciam a imagem corporal dos adolescentes, ficando especialmente vulneráveis durante estas alterações do corpo. A precocidade ou retardamento destas transformações físicas, a comparação com os pares, a forma como a família, os professores e os colegas lidam com a situação tem impacto na aceitação do corpo dos púberes e na forma como lidam com o corpo na adolescência.

Devido às discrepâncias das experiências que separam os adolescentes mais jovens dos mais velhos, a UNICEF (2011) sugere uma divisão da adolescência: uma fase inicial 
que ocorre entre os 10 e os 14 anos e uma fase final da adolescência que ocorre entre os 15 e os 19 anos de idade. Na perspetiva de Wong et al. (2011) a adolescência ocorre em três fases distintas: um início da adolescência (11-14 anos), uma adolescência média (15-17 anos) e um fim da adolescência (18-20 anos). A divisão do período de adolescência em fases não é unanime quanto ao número nem quanto ao período etário que compreendem. O estudo da adolescência não pode ser independente do efeito de coorte, das variações históricas e sociológicas (Rodrigues et al., 2013) e, ainda, dos contextos culturais.

As reações à forma, tamanho ou aparência real/aparência imaginada, podem variar dependendo da idade, sexo, orientação sexual, cultura, prática desportiva, amigos, família, influência dos meios de comunicação social, estilos de vida geral, entre outras variáveis (Alebachew \& Ashagrie, 2017; Cash, 2008; Gay, 2009; Grogan, 2017; Smolak, 2012). A imagem corporal é diferenciada em função do género dos adolescentes havendo maior incidência nas raparigas sobre a perceção de imagem corporal mais negativa (Rodrigues et al., 2013).

Segundo Nogueira et al. (2010), a insatisfação e distorção da imagem corporal dos adolescentes está relacionada com as transformações físicas e com a exigência de um padrão estético que valoriza a magreza, particularmente na mulher. A insatisfação corporal focaliza claramente as preocupações com o peso, forma do corpo e gordura corporal. A ideia de um corpo magro, atlético e esbelto não é indissociada do consumo, tendo em conta a oferta de produtos e serviços num mercado crescente (Carvalho et al., 2005) e da influência dos media e redes sociais podem influenciar negativamente a imagem corporal (Lonergan et al., 2019).

$\mathrm{Na}$ adolescência subsistem sentimentos desconfortáveis com as alterações corporais, e à medida que os adolescentes começam a adquirir imagens de si mesmo, deparam-se com discrepâncias entre os seus pares (Wong et al., 2011). Para estes autores, o autoconceito torna-se mais diferenciado na medida em que vão adquirindo uma imagem mais complexa de si mesmos. Wehrle e Fasbender (2019) defendem o autoconceito como uma estrutura multidimensional, multifacetada e dinâmica, permitindo que o indivíduo processe informações relevantes, em que a autoimagem considerada central na vida subjetiva, influencia os pensamentos, sentimentos e os comportamentos que um indivíduo tem sobre si (Rosenberg, 1965). Já em 1968 Rosen e Ross, demonstraram num estudo que a satisfação com a imagem corporal e a satisfação com o autoconceito se relacionam positivamente.

As evidências sobre a importância da construção de uma imagem corporal positiva durante o período da adolescência, levam a que se tenha em consideração as melhores práticas para o desenvolvimento e validação de escalas de medida destinadas para os aspetos 
sociais, comportamentais e da saúde (Boateng et al., 2018). A adaptação das escalas CARSAL e CARVAL para adolescentes, revela-se pertinente para este período do desenvolvimento humano, no qual ocorrem as maiores transformações na imagem corporal e aparência (Gay, 2009; Grogan, 2017; Medeiros, 2013; Rumsey \& Harcourt, 2012; Wong et al., 2011).

\section{MÉTODO}

O presente estudo é descritivo, psicométrico, quantitativo, transversal e exploratório.

\subsection{Participantes}

A amostra do estudo é constituída por 196 adolescentes. Os participantes identificam-se como sendo do sexo feminino e masculino, com idades compreendidas entre os 11 e os 17 anos $(M=15.05 ; D P=1.62)$. Em termos de escolaridade, frequentavam o $2^{\circ}$ ciclo $(n=11)$, o $3^{\circ}$ ciclo $(n=77)$, e o secundário $(n=103)$, nas ilhas de São Miguel e do Faial na Região Autónoma dos Açores. A maioria dos participantes é do sexo feminino $(n=127 ; 66.1 \%)$.

\subsection{Instrumentos}

Em função dos objetivos optou-se pela seleção e aplicação de três instrumentos. Assim, procedeu-se à realização de um Questionário Sociodemográfico, no intento de explorar as variáveis: idade, sexo, nível de escolaridade, habilitações académicas dos pais e autorreferenciação de algum reconhecimento ou diferença visível na face (e.g., acne, cicatriz, manchas). Optou-se, igualmente, pela aplicação das escalas CARSAL e CARVAL, e da escala de Avaliação do Autoconceito de Adolescentes (versão do Piers-Harris).

As escalas CARSAL e CARVAL [Center for Appearance Research Salience Scale and Center for Appearance Research Valence Scale (CARSAL/CARVAL), da autoria de Moss e Rosser, (2012), traduzida e adaptada por Mendes et al. (2019)], avaliam dois aspetos do auto esquema da aparência: a saliência e a valência. A saliência é a medida em que a informação da aparência auto relevante é trazida à consciência e a valência é a avaliação emocional do self em relação à aparência. As escalas CARSAL e CARVAL são compostas por 13 itens de autorresposta, de formato tipo Likert, com uma ponderação de 6 pontos, variando a cotação entre 1 (Discordo totalmente) e 6 (Concordo totalmente). Em estudos anteriores com uma amostra na população portuguesa adulta (Mendes et al., 2019), a confiabilidade interna, pela determinação do alfa de Cronbach, para a escala CARSAL para 
uma amostra em adultos portugueses foi de $\alpha=.77$, enquanto a escala CARVAL foi de $\alpha=$ .93 .

A escala de Avaliação do Autoconceito de Adolescentes (versão do Piers-Harris) reduzida a 30 itens em escala 1-6 [Piers-Harris Children's Self-Concept Scale, da autoria de Piers e Herzberg (2002) e adaptada por Veiga e Leite (2016)], envolve aspetos específicos do autoconceito. A escala em referência é composta por seis fatores: Ansiedade, Aparência Física, Comportamento, Popularidade, Felicidade, e Estatuto Intelectual. A escala é composta por 30 itens, de tipo Likert, com seis opções de resposta (desde 1 - Discordo totalmente a 6 - Concordo totalmente). A confiabilidade interna da escala foi de $\alpha=.87$.

\subsection{Procedimentos}

Em se tratando de uma amostra de adolescentes inseridos no sistema escolar público, solicitou-se à Direção Regional da Educação da Região Autónoma dos Açores, autorização para a realização do presente estudo nas escolas básicas e secundárias das ilhas de São Miguel e do Faial. A escolha destas ilhas deve-se a amostra de conveniência dos investigadores. Uma vez que as unidades orgânicas do sistema educativo regional, gozam de autonomia pedagógica e administrativa, solicitou-se a cada presidente do conselho executivo das escolas do contexto geográfico referido, o pedido de autorização para a realização do presente estudo.

Por se tratar de uma amostra de menores de idade, procedeu-se ao pedido de autorização aos respetivos encarregados de educação dos estudantes adolescentes, e obtevese a anuência destes através de um consentimento livre e esclarecido, permitindo aos adolescentes a total liberdade de participar no estudo e responder aos instrumentos apresentados em papel. Foi assegurada a confidencialidade e anonimato de todas as respostas. O estudo seguiu as linhas de orientação e princípios éticos propostos pela American Psychology Association (APA) quando a investigação envolve seres humanos (APA, 2020).

Para avaliar a qualidade das afirmações apresentadas nas escalas CARSAL e CARVAL, realizou-se um pré-teste a vinte participantes, subdivididos em dois subgrupos, sendo um mais centrado na pré-adolescência, com idades compreendidas entre os 10 e os 14 anos $(n=10)$, e o outro mais centrado na adolescência, propriamente dita, e com idades entre os 15 e os 17 anos $(n=10)$. A não verificação de dúvidas na compreensão dos itens da escala 
CARSAL e CARVAL permitiu a aplicação do respetivo protocolo de investigação em várias escolas da Ilha de São Miguel e Ilha do Faial.

Atendendo a que a adaptação das escalas CARSAL e CARVAL numa amostra de adultos portugueses incluiu a idade mínima 18 anos, decidiu-se que para o presente estudo, focado nos adolescentes, a idade máxima dos participantes seria 17 anos de idade. Dos 200 participantes iniciais foram eliminados 4 por não cumprirem os critérios de inclusão, isto é, preenchimento de todas as questões dos instrumentos de recolha de dados e idade situada no intervalo entre os 10 e os 17 anos.

\subsection{Análise Estatística}

Para os procedimentos estatísticos recorreu-se ao software SPSS, na versão 27 para o sistema operativo macOS e ao software AMOS, na versão 27 para o sistema operativo Windows. Procedeu-se a uma análise fatorial exploratória (AFE) e verificou-se a fiabilidade das medidas, através da determinação do coeficiente alfa de Cronbach $(\alpha)$, no intuito de avaliar a correlação entre os itens, de forma a aferir a medição do constructo teórico. Posteriormente, ao analisar-se que a distribuição da amostra não respeita a normalidade, efetuou-se a análise do coeficiente de correlação Spearman $\left(R_{S}\right)$ para quantificar a intensidade e a direção da associação entre as dimensões dos instrumentos (escalas CARSAL e CARVAL e escala de Avaliação do Autoconceito de Adolescentes, versão de Piers-Harris).

Posteriormente, determinou-se a análise fatorial confirmatória (AFC), com a estimação de máxima verosimilhança, avaliando a qualidade de ajustamento global do modelo fatorial pelo teste Qui-quadrado de Ajustamento $\left(X^{2}\right)$, considerando $X^{2} / g . l$ inferior a 5 como modelo aceitável. Tendo como referência os índices Comparative Fit Index (CFI), Goodness of Fit Index (GFI), Ticher-Lewis Index (TLI), Root Mean Square Error of Approximation (RMSEA), considera-se um bom ajustamento quando os índices são próximos do valor de 1 e o RMSEA apresenta valores de referência inferiores a .05 . A fiabilidade compósita (FC) e a variância extraída média (VEM) foram avaliadas através das respetivas fórmulas (Marôco, 2014).

\section{RESULTADOS}

\subsection{Fiabilidade}

A versão das escalas CARSAL (saliência) e CARVAL (valência), composta por 13 itens, e aplicada à amostra de adolescentes já referida, apresenta um índice de consistência interna de $\alpha=.76$ para a dimensão Saliência (5 itens) e $\alpha=.93$ para a dimensão Valência (8 itens). 
Verificou-se a intensidade da associação entre a Escala CARSAL/CARVAL e a Escala de Avaliação do Autoconceito de Adolescentes (versão do Piers-Harris) e respetivas dimensões (Tabela 1). Os resultados evidenciam elevados níveis de associação negativa entre a escala CARVAL, o Autoconceito Total e duas dimensões do Autoconceito (e.g., Aparência Física e Felicidade).

Tabela 1

Correlação entre as escalas CARSAL e CARVAL, e a Escala de Avaliação do Autoconceito de Adolescentes versão de Piers-Harris

\begin{tabular}{|c|c|c|c|c|c|c|c|c|c|}
\hline & 1 & 2 & 3 & 3.1 & 3.2 & 3.3 & 3.4 & 3.5 & 3.6 \\
\hline 2. CARVAL (valência) & & -- & $-.730 * * *$ & $-.363 * * *$ & $-.773 * * *$ & $-.255^{* * * *}$ & $-.467 * * *$ & $-.618 * * *$ & $-.471 * * *$ \\
\hline 3. Autoconceito Total & & & -- & $.637 * * *$ & $.754 * * *$ & $.424 * * *$ & $.703 * * *$ & $.750 * * *$ & $.676^{* * *}$ \\
\hline 3.1 Ansiedade & & & & -- & $.294 * * *$ & .112 & $.480 * * *$ & $.417 * * *$ & $.159 *$ \\
\hline 3.4 Popularidade & & & & & & & -- & $.452 * * *$ & $.411 * * *$ \\
\hline 3.5 Felicidade & & & & & & & & -- & $.403 * * *$ \\
\hline 3.6 Estado Intelectual & & & & & & & & & -- \\
\hline
\end{tabular}

Encontrou-se, ainda, níveis de intensidade moderada e negativa entre a escala CARVAL e três das dimensões do Autoconceito (e.g., Ansiedade, Popularidade e Estado Intelectual). Obtiveram-se níveis de associação mais fraca e negativa entre a escala CARVAL e a dimensão Comportamental do Autoconceito. Verifica-se que a Escala CARSAL somente apresenta uma correlação fraca e negativa com a dimensão Ansiedade e uma correlação fraca e positiva com a dimensão Aparência Física.

\subsection{Análise Fatorial Exploratória}

Procedeu-se ao método de análise de componentes principais, com rotação Varimax, tendose verificado os itens das escalas CARSAL e CARVAL. A medida de Kaiser-Meyer-Olkin (KMO) avalia a qualidade da adequação da Análise de Componentes Principais (ACP), em que os valores superiores a .80 se consideram muito bons $(\mathrm{KMO}=.89)$. Através do teste de esfericidade de Bartlett $\left(X^{2}(78)=1600.43 ; p=<.001\right)$, verifica-se que as variáveis estão suficientemente correlacionadas a ponto de ser apropriado realizar uma análise fatorial 
exploratória. A Figura 1 apresenta a regra do eigenvalue superior a 1 e com o Scree Plot, a estrutura relacional é explicada por dois fatores que explicam $68 \%$ da variabilidade total.

\section{Figura 1}

Critério do Scree Plot para as escalas CARSAL e CARVAL

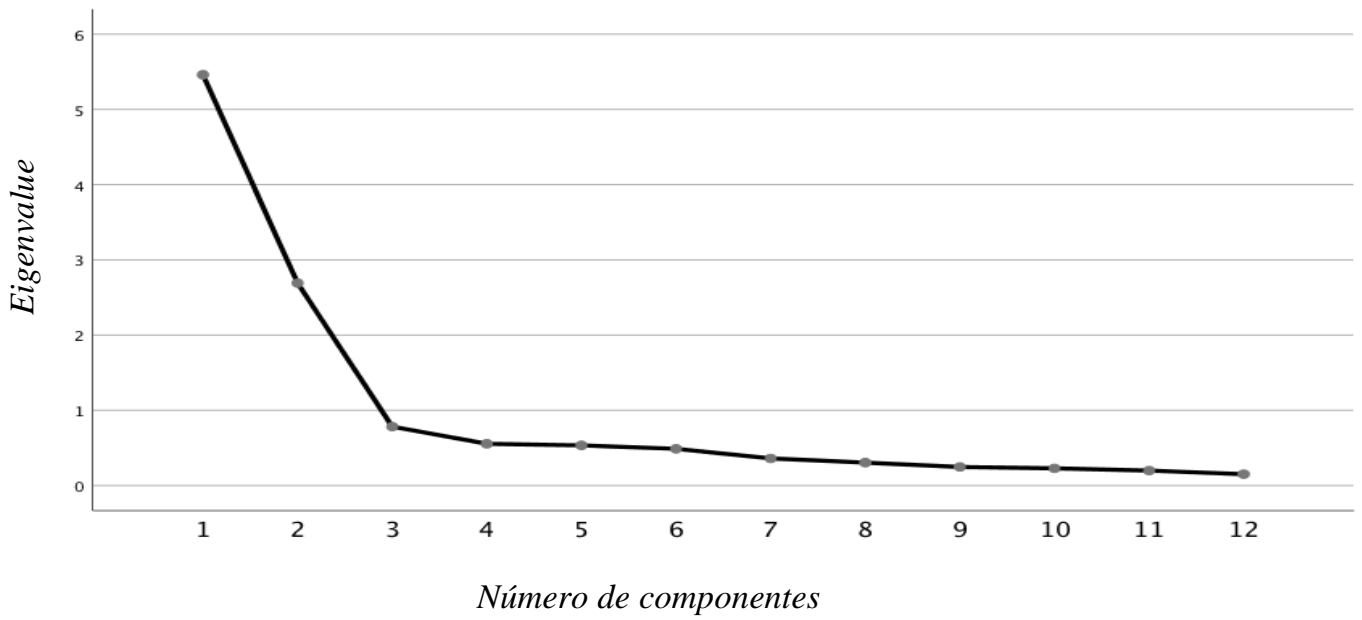

Nota: Center for Appearance Research Salience Scale (CARSAL); Center for Appearance Research Valence Scale (CARVAL)

Constata-se que as correlações item-total nas escalas CARSAL e CARVAL aplicada numa amostra de adolescentes, variam entre .57 e .89 , verificando-se correlações moderadas a elevadas na maioria dos itens (Tabela 2).

\section{Tabela 2}

Comparação das Correlações do Item-Total das Escalas CARSAL e CARVAL Versão Adultos e Versão Adolescentes de Piers-Harris

\begin{tabular}{|c|c|c|c|}
\hline Item & Descrição do Item & $\begin{array}{l}\text { Correlação: Item-Total } \\
\text { Versão Adultos }(r)\end{array}$ & $\begin{array}{l}\text { Correlação: Item-Total } \\
\text { Versão Adolescentes }(r)\end{array}$ \\
\hline 1 & $\begin{array}{l}\text { Para mim, a minha aparência é uma parte } \\
\text { importante do que eu sou. }\end{array}$ & .527 & .645 \\
\hline 2 & $\begin{array}{l}\text { Muitas vezes tenho consciência da maneira como } \\
\text { sou olhado pelas outras pessoas. }\end{array}$ & .767 & .717 \\
\hline 3 & $\begin{array}{l}\text { Na maioria das situações, dou por mim a ter } \\
\text { consciência da maneira como a minha face e corpo } \\
\text { parecem. }\end{array}$ & .846 & .767 \\
\hline 4 & $\begin{array}{l}\text { Penso muitas vezes no impacto que a aparência do } \\
\text { meu corpo e face transmitem. }\end{array}$ & .820 & .816 \\
\hline 5 & Geralmente estou consciente da minha aparência. & .614 & .575 \\
\hline 6 & Estou satisfeito(a) com a minha aparência física. * & .870 & .873 \\
\hline 7 & Não gosto da minha aparência. & .807 & .855 \\
\hline 8 & $\begin{array}{l}\text { A minha aparência faz-me sentir bem comigo } \\
\text { mesmo(a).* }\end{array}$ & .881 & .888 \\
\hline 9 & A minha aparência é pouco atraente. & .755 & .621 \\
\hline 10 & $\begin{array}{l}\text { O meu corpo e a minha face parecem tão bem como } \\
\text { eu gostaria.* }\end{array}$ & .763 & .847 \\
\hline 11 & $\begin{array}{l}\text { Sinto-me mal com o meu corpo e a minha } \\
\text { aparência. }\end{array}$ & .784 & .873 \\
\hline 12 & Gosto da minha aparência. * & .902 & .891 \\
\hline 13 & A minha aparência faz-me sentir atraente. * & .894 & .740 \\
\hline
\end{tabular}

Nota: * itens invertidos; Center for Appearance Research Salience Scale (CARSAL); Center for Appearance Research Valence Scale (CARVAL) 
Verificaram-se relações idênticas à validação e adaptação das escalas CARSAL e CARVAL para uma amostra de adultos portuguesa.

\subsection{Análise Fatorial Confirmatória}

A estrutura das dimensões das escalas CARSAL e CARVAL numa amostra de adolescentes apresenta índices de qualidade de ajustamento considerados sofríveis $\left(X^{2} / \mathrm{df}=4.10 ; \mathrm{CFI}=\right.$ .87 ; GFI $=.83 ;$ TLI $=.85 ;$ RMSEA $=.127 ; p[$ rmsea $\leq .05]=<.001)$. A regressão e as variâncias de todos os itens são significativas $(p \leq .001)$. Tendo-se verificado um ajustamento considerado sofrível e verificado que a raiz quadrada da matriz dos erros (RMSR $=.003$ ) revela que o ajustamento se considera bom, procedeu-se à refinação do modelo pelas maiores covariâncias apresentadas pelo índice de modificação obtido. Confrontando a Figura 2, verifica-se que a reespecificação do modelo pela covariância dos erros de medida nos itens $7,8,9,10,11$ e 13, permitiu a verificação de um melhor ajustamento $\left(X^{2} / \mathrm{df}=3.377\right.$; $\mathrm{GFI}=.88 ; \mathrm{CFI}=.91 ; \mathrm{TLI}=.87 ; \mathrm{RMSEA}=.11 ; p[\mathrm{rmsea} \leq .05]=<.001)$, no entanto, Marôco (2018) menciona que o modelo ao apresentar um valor de RMSEA superior a .1, o modelo não se pode aceitar.

\section{Figura 2}

Modelo Modificado de Análise Fatorial Confirmatória das escalas CARSAL e CARVAL ( $n=192)$.

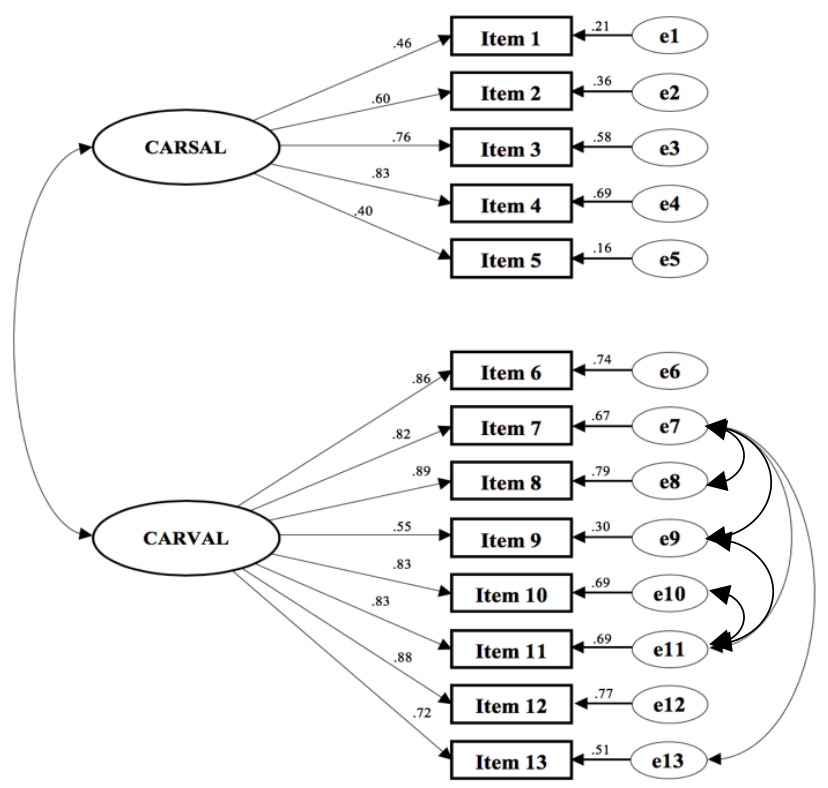

Assim, pelos pesos fatoriais estandardizados, calculou-se a fiabilidade compósita, que se revelou apropriada quer para a subescala CARSAL $(\widehat{F C}=.76)$, quer para a subescala CARVAL $(\widehat{F C}=.93)$. A variância extraída média, um indicador da validade convergente 
dos fatores, revelou-se baixo (próximo do aceitável) para a subescala CARSAL $(\widehat{V E M}=$ $.40)$, e indicador de validade convergente adequada para a subescala CARVAL $(\widehat{V E M}=$ $.64)$.

As escalas CARSAL e CARVAL, apresentam uma correlação reduzida $(r=.003 ; p$ $=.97)$, verificando-se que a escala CARSAL apresenta uma validade convergente considerada baixa. Assim, procedeu-se a uma análise fatorial confirmatória para esta escala. O modelo da escala CARVAL constituído por oito itens, apresenta uma melhor qualidade de ajustamento $\left(X^{2} / \mathrm{df}=4.32 ; \mathrm{CFI}=.95 ; \mathrm{GFI}=.89 ; \mathrm{TLI}=.92 ; \mathrm{RMSEA}=.132 ; p[\mathrm{rmsea} \leq\right.$ $.05]=<.001)$, todavia, através do valor RMSEA verifica-se que o modelo também não se considera aceitável.

Figura 3

Modelo Modificado de Análise Fatorial Confirmatória da CARVAL $(n=192)$.

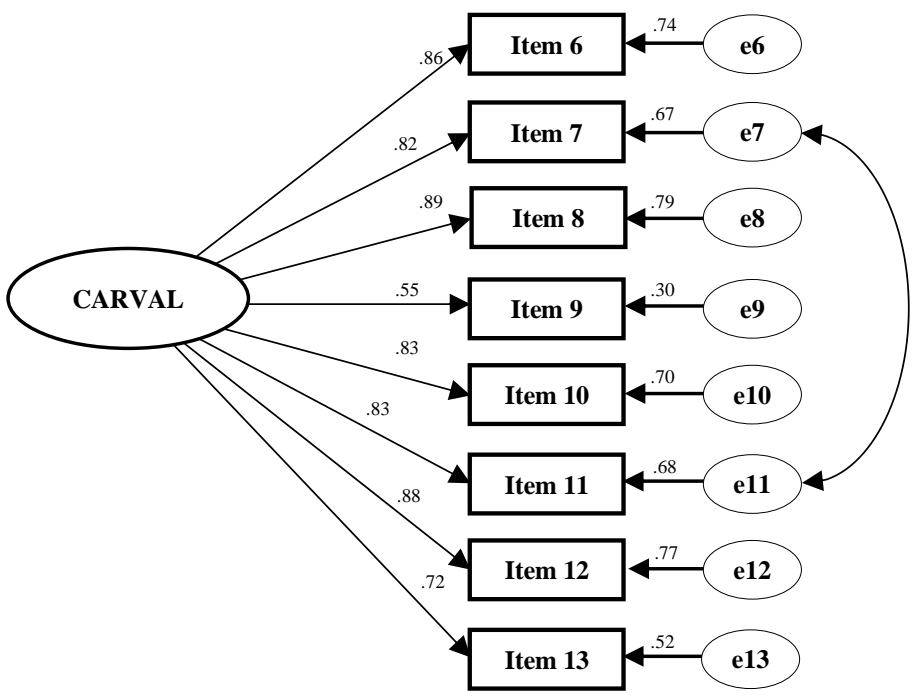

Através da reespecificação do modelo CARVAL pela covariância dos erros de medida nos itens 7 e 11 (cf., Figura 3), foi possível obter um modelo considerado aceitável $\left(X^{2} / \mathrm{df}=2.59 ; \mathrm{CFI}=.98 ; \mathrm{GFI}=.95 ; \mathrm{TLI}=.96 ; \mathrm{RMSEA}=.09 ; p[\mathrm{rmsea} \leq .05]=.017\right)$.

\subsection{Análise de Regressões}

O pressuposto da independência foi validado com a estatística de Durbin-Watson $(d=1.86)$ como descrito em Marôco (2018). A regressão linear múltipla permitiu identificar que somente a escala CARVAL $(\beta=-.734, t(190)=-14.91 ; p=<.001)$ se considera como preditor significativo. O modelo significativo, prevê que a escala CARVAL representa $53.7 \%$ da variabilidade na variável dependente. 


\subsection{Comparação de Médias}

A realização de teste Wilcoxon-Mann-Whitney não verificou diferenças estatisticamente significativas entre os períodos de adolescência. Verificaram-se diferenças significativas entre a variável Sexo e a variável escala CARSAL, observando-se diferenças estatisticamente significativas $\left(U=3,029.00 ; p_{U}=.003 ; \mathrm{N}=192\right)$, com um TDE considerado pequeno $d$ de Cohen $=.22$ (Espírito-Santo \& Daniel, 2015).

Analisadas diferenças entre a variável Ter uma Diferença Visível (e.g. cicatriz, acne, outro) na face e as variáveis escala CARSAL (até que ponto a aparência e o eu físico são trazidos à perceção da consciência) e escala CARVAL (avaliação da aparência de maneira positiva/negativa), através do teste Wilcoxon-Mann-Whitney observaram-se diferenças estatisticamente significativas $\left[U_{\text {CARSAL }}=3,578.50 ; \mathrm{p}=.009 ; d\right.$ de Cohen $=.19 ; \mathrm{N}=192$ ); $\left(U_{\text {CARVAL }}=3,720.50 ; \mathrm{p}=.025 ; d\right.$ de Cohen $\left.\left.=.16 ; \mathrm{N}=192\right)\right]$ com um TDE considerado pequeno para ambas as variáveis.

Os resultados da realização do teste Kurskal-Wallis comprovaram a não verificação de diferenças estatísticas e significativas entre as restantes variáveis sociodemográficas (e.g., nível de escolaridade dos adolescentes, habilitações dos pais) e respetivas escalas CARSAL e CARVAL.

\section{DISCUSSÃO}

As escalas CARSAL e CARVAL são um instrumento aferido por Moss e Rosser (2012) e avaliam dois aspetos dos auto-esquemas da aparência (Saliência e Valência), tendo sido adaptado para a população portuguesa adulta. $\mathrm{O}$ presente estudo psicométrico apresenta a validação das escalas CARSAL e CARVAL para uma amostra de adolescentes portugueses, residentes na Região Autónoma dos Açores.

Os resultados relativos ao índice de consistência interna, demonstram-se moderados para a escala CARSAL $(\alpha=.77)$ e uma elevada consistência interna para a escala CARVAL $(\alpha=.93)$. Estes valores apresentam-se idênticos à versão portuguesa adaptada numa amostra de adultos portugueses (Mendes et al., 2019). A medida de adequação da amostragem KMO, verificou uma boa homogeneidade das variáveis (Marôco, 2018, p. 467). Através da Tabela 2, é possível verificarem-se correlações fortes, a muito fortes para os itens da escala CARSAL $(r>.50 ; r<.90)$ e escala CARVAL $(r>.60 ; r<.90)$. Considerando-se o critério de Kaiser (regra do eigenvalue superior a 1) e o critério do Scree Plot, foi possível determinar 
dois fatores no modelo fatorial, conforme a escala original e respetiva validação/adaptação para Portugal, numa amostra de adultos.

O primeiro modelo de equação estrutural testado, através da análise fatorial confirmatória, evidenciou índices de qualidade do ajustamento sofrível, no entanto, a reespecificação do modelo pelos erros estimados de maior covariância, permitiu obter um melhor nível de qualidade de ajustamento. Marôco (2014) menciona que uma fiabilidade compósita superior a .70, é considerada indicador de uma fiabilidade de constructo apropriada, tendo-se verificado no presente estudo, que a escala CARSAL $(\widehat{F C}=.76)$ e a escala CARVAL $(\widehat{F C}=.93)$, apresentam indicadores de uma fiabilidade de constructo adequados. No entanto só se verificou validade convergente apropriada para a escala CARVAL e valores próximos de validade convergente para a escala CARSAL. Estes dados também se revelaram idênticos à adaptação e validação das escalas CARSAL e CARVAL para a amostra de adultos portugueses (Mendes et al., 2019), podendo as características da amostra explicarem as debilidades relacionadas com as cargas fatoriais do instrumento CARSAL/CARVAL para a presente amostra de adolescentes.

Smolak (2012) demonstrou que as preferências e as preocupações com a aparência, principalmente a face e a fisionomia corporal são evidentes nos primeiros anos da infância, exercem um impacto no desenvolvimento do self. No presente estudo, a escala CARSAL (perceção consciente da aparência e do eu físico) não revelou uma associação com o autoconceito dos participantes, podendo estes resultados deverem-se ao facto de somente uma parte do autoconhecimento estar ativa na memória. Estes resultados podem revelar que os adolescentes apresentam uma baixa autoconsciência da sua aparência (Moss et al., 2014), em que os adolescentes sentem insegurança da imagem corporal que ainda está em construção. No entanto, constatou-se que os participantes em estudo apresentam uma forte associação negativa entre a valência (CARVAL), Autoconceito Total e duas dimensões do Autoconceito (e.g., Aparência Física e Felicidade) apresentando correlações moderadas a fracas para as restantes dimensões (Marôco, 2018, p. 23). Estas correlações podem dever-se ao facto de existir uma associação entre a imagem corporal e o autoconceito (Rosen \& Ross, 1968) em que a construção da imagem corporal contribuiu para a perceção do autoconceito do indivíduo (Falconi et al., 2019).

Estudos (e.g., Gay, 2009; Almeida et al., 2005; Grogan, 2017; Rodrigues et al., 2013) revelam que o sexo feminino apresenta uma tendência, a preocupar-se mais com o seu corpo quando comparado com o sexo masculino. Na perspetiva de Ferreira et al. (2018) essas 
diferenças referem-se à escassa investigação sobre as preocupações com a imagem corporal com participantes do sexo masculino, facto que não podemos descurar. Contudo, o presente estudo verificou que o sexo feminino apresenta maior autoconsciência da aparência (escala CARSAL) quando comparadas com o sexo masculino. De facto, estudos defendem que o sexo feminino apresenta maior consciência da sua imagem corporal, em relação ao sexo oposto (Javaid \& Ajmal, 2019; Kumar, 2016; Levine \& Smolak, 2004; Moss et al., 2014; Quittkat et al., 2019).

Verificaram-se diferenças entre os adolescentes com uma diferença visível na face (e.g., cicatriz, acne, manchas, outros), sobressaindo uma avaliação mais negativa da sua aparência, no qual o eu físico é trazido à perceção da consciência da aparência, quando comparados com adolescentes sem uma diferença visível na face. Rumsey e Harcourt (2007) revelam que os adolescentes que vivem com uma diferença visível têm uma perceção mais negativa de si próprios e têm medo das avaliações de seus pares, podendo resultar em problemas comportamentais e psicossociais. Recentemente, uma revisão sistemática da literatura demonstra que os adolescentes que vivem com uma diferença visível (desfiguramento) apresentam maiores níveis de ansiedade (van Dalen et al., 2020).

As limitações deste estudo prendem-se, essencialmente, com duas ordens de fatores: pelo facto de a amostra de adolescentes ser integralmente de duas ilhas da Região Autónoma dos Açores (Faial e São Miguel), e por não terem sido aplicados outros instrumentos que avaliassem outras variáveis relacionadas com a avaliação da imagem corporal como, por exemplo, o índice de massa corporal e a imagem corporal positiva.

\section{CONCLUSÕES}

Durante o período da adolescência o indivíduo depara-se com mudanças significativas na imagem corporal, em que o autoconceito, classificado em diversos tipos (académico, emocional, social ou físico) ajudam a compreender aspetos importantes do indivíduo (e.g., noção de identidade) revelando-se um processo dinâmico. As escalas CARSAL e CARVAL revela-se um instrumento pertinente na contribuição de um maior conhecimento sobre como os adolescentes envolvem os esquemas corporais na perceção da imagem corporal. Apesar de o modelo reespecificado das escalas CARSAL e CARVAL não se apresentar tão robusto como a adaptação e validação numa amostra de adultos, a escala CARVAL apresenta-se robusta na avaliação positiva ou negativa que os adolescentes fazem da sua aparência. 
Apesar deste estudo abrir potencialidades na medida de avaliação da imagem corporal de adolescentes, a amostra foi reduzida e circunscrita a um contexto insular e de apenas duas ilhas, limitações que se assinalam. Estudos futuros devem ser realizados com a inclusão de adolescentes de outras regiões de Portugal Continental e Ilhas (Região Autónoma da Madeira) e explorar outras variáveis psicológicas, tais como os traços de personalidade, o otimismo, a autoestima, as quais possam influenciar a construção de auto esquemas da aparência positivos.

Conflitos de interesse: Os autores declaram a inexistência de conflitos de interesse na realização do trabalho. Os autores participam na equipa Editorial, mas não desempenharam qualquer papel de intervenção no processo de revisão por pares double-blinded.

Fontes de financiamento: Não existem fontes externas de financiamento para a realização deste artigo.

Agradecimentos: Os autores não têm quaisquer apoios a declarar.

\section{Referências}

Alebachew, F., \& Ashagrie, M. (2017). The body-image concept analysis of youth and adolescent. American Journal of Biomedical and Life Sciences, 5(6), 130-134. https://doi.org/10.11648/j.ajbls.20170506.14

Almeida, G. A. N., Santos, J. E., Pasian, S. R., \& Loureiro, S. (2005). Perceção de tamanho e forma corporal de mulheres: Estudo exploratório. Psicologia em Estudo, 10(1), 27-35. http://www.scielo.br/pdf/pe/v10n1/v10n1a04.pdf.

APA. (2020). Publication manual of the American Psychological Association (7th ed.). American Psychological Association. https://doi.org/10.1037/0000165-000

Boateng, G. O., Neilands, T. B., Frongillo, E. A., Melgar-Quiñonez, H. R., \& Young, S. L. (2018). Best practices for developing and validating scales for health, social, and behavioral research: A primer. Frontiers in Public Health, 6, 1-18. https://doi.org/10.3389/fpubh.2018.00149

Carvalho, A. M. P., Cataneo, C., Galindo, E. M. C., \& Malfará, C. T. (2005). Autoconceito e imagem corporal em crianças obesas. Paidéia, 15(30), 131-139. https://www.scielo.br/j/paideia/a/TQcpJhvdv3S45cZxZ7FFRZP/?format=pdf\&lang $=\mathrm{pt}$.

Cash, T. F. (2006). The influence of sociocultural factors on body image: Searching for constructs. Clinical Psychology: Science and Practice, 12(4), 438-442. https://doi.org/10.1093/clipsy.bpi055

Cash, T. F. (2008). The body image workbook: An eight-step program for learning to like your looks (2nd ed.). New Harbinger Publications.

Espírito-Santo, H., \& Daniel, F. B. (2015). Calcular e apresentar tamanhos do efeito em trabalhos científicos (1): As limitações do $\mathrm{p}<0,05$ na análise de diferenças de médias de dois grupos. Revista Portuguesa de Investigação Comportamental e Social, 1(1), 3-16. https://doi.org/10.7342/ismt.rpics.2015.1.1.14 
Falconi, C. A., Zanetti, M. C., Santos, T. de A., Dias, H. M., Brandao, M. R. F., \& Neves, A. N. (2019). Relação entre antropometria, gordura corporal e autoconceito de adolescentes do sexo feminino. Cuadernos de Psicología del Deporte, 19(2), 256-264. https://doi.org/10.6018/cpd.348001

Ferreira, C., Marta-Simões, J., Oliveira, S., \& Duarte, J. (2018). Estudo da estrutura fatorial e das qualidades psicométricas da versão portuguesa da Male Body Attitude Scale-Revised. Revista Portuguesa de Investigação Comportamental e Social, 4(2), 16-24. https://doi.org/10.31211/rpics.2018.4.2.74

Gay, K. (2009). Body image and appearance: The ultimate teen guide. Scarecrow Press.

Grogan, S. (2017). Body image: Understanding body dissatisfaction in men, women and children (3rd ed.). Routledge.

Hockenberry, M. J., Wilson, D., \& Wong, D. L. (2013). Wong's essentials of pediatric nursing (9th ed.). Elsevier.

Javaid, Q. A., \& Ajmal, A. (2019). The impact of body image on self-esteem in adolescents. Clinical and ounselling Psychology Review, 1(1), 44-54. https://doi.org/10.32350/ccpr.11.04

Kumar, H. (2016). Gender difference regarding body image: A comparative study. Advances in Obesity, Weight Management \& Control, 4(4). https://doi.org/10.15406/aowmc.2016.04.00092

Levine, M., \& Smolak, L. (2004). Body image development in adolescence. In T. Cash \& T. Pruzinsky (Eds.), Body image: A handbook of theory, research, and clinical practice (2sd Ed., pp. 74-82). Guilford Press.

Lonergan, A. R., Bussey, K., Mond, J., Brown, O., Griffiths, S., Murray, S. B., \& Mitchison, D. (2019). Me, my selfie, and I: The relationship between editing and posting selfies and body dissatisfaction in men and women. Body Image, 28, 39-43. https://doi.org/10.1016/j.bodyim.2018.12.001

Maciel, N., \& Rebelo, Ó. (2013). Da puberdade à adolescência: Desenvolvimento físico, fisiológico e sexual. In T. Medeiros (Coord.), Adolescência: Desafios e Riscos. Letras Lavadas.

Marôco, J. (2014). Análise de Equações Estruturais: Fundamentos teóricos, software \& aplicações (2th ed.). ReportNumber.

Marôco, J. (2018). Análise Estatística com o SPSS Statistics (7th ed.). Report Number.

Medeiros, T. (2013). Adolescência: Desafios e Riscos. Letras Lavadas.

Mendes, J., Rego, R., \& Pereira, V. (2019). Tradução e adaptação da escala CARSAL/CARVAL para Portugal: Estudo psicométrico. PSICOLOGIA, 33(1), 47-54. https://doi.org/10.17575/rpsicol.v33i1.1425

Moss, T. P., \& Rosser, B. A. (2012). The moderated relationship of appearance valence on appearance self consciousness: Development and testing of new measures of appearance schema components. PLoS ONE, 7(11), 1-7. https://doi.org/10.1371/journal.pone.0050605

Moss, T., Lawson, V., White, P., \& The Appearance Research Collaboration. (2014). Salience and valence of appearance in a population with a visible difference of appearance: Direct and moderated relationships with self-Consciousness, anxiety and depression. PLoS ONE, 9(2), 1-8. https://doi.org/10.1371/journal.pone.0088435

Nogueira, S. G., Macedo, V. S., \& Guedes, P. M. (2010). Avaliação da imagem corporal e de comportamentos alimentares como possíveis desencadeadores de transtornos alimentares em bailarinas pré-adolescentes. Nutrir Gerais, 4(6), 538-553. https://docplayer.com.br/5435403-Avaliacao-da-imagem-corporal-e-de-comportamentosalimentares-como-possiveis-desencadeadores-de-transtornos-alimentares-em-bailarinaspre-adolescentes.html. 
Papalia, D. E., \& Feldman, R. D. (2013). Desenvolvimento humano (12th ed.). MacGraw Hill Artemed.

Piers, E., \& Herzberg, D. (2002). Piers-Harris Children's Self-Concept Scale (2th ed.). Western Psychological Services.

Pitron, V., \& De Vignemont, F. (2017). Beyond differences between the body schema and the body image: Insights from body hallucinations. Consciousness and Cognition, 53, 115-121. https://doi.org/10.1016/j.concog.2017.06.006

Quittkat, H. L., Hartmann, A. S., Düsing, R., Buhlmann, U., \& Vocks, S. (2019). Body dissatisfaction, importance of appearance, and body appreciation in men and women over the lifespan. Frontiers in Psychiatry, 10, 1-12. https://doi.org/10.3389/fpsyt.2019.00864

Ricciardelli, L. A., \& Yager, Z. (2016). Adolescence and body image: From development to preventing dissatisfaction. Routledge, Taylor \& Francis Group.

Rodrigues, D., Teves, C., \& Medeiros, T. (2013). Autoimagem e satisfação corporal na adolescência. In T. Medeiros (Coord.), Adolescência: Desafios e riscos (pp. 183209). Letras Lavadas.

Rosen, G. M., \& Ross, A. O. (1968). Relationship of body image to self-concept. Journal of Consulting and Clinical Psychology, 32(1), 100-100. https://doi.org/10.1037/h0025433

Rosenberg, M. (1965). Society and the adolescent self-image. Princeton University Press.

Rumsey, N., \& Harcourt, D. (2007). Visible difference amongst children and adolescents: Issues and interventions. Developmental Neurorehabilitation, 10(2), 113-123. https://doi.org/10.1080/13638490701217396

Rumsey, N., \& Harcourt, D. (Eds.). (2012). The Oxford handbook of the psychology of appearance. Oxford University Press.

Smolak, L. (2012). Appearance in chilhood and adolescence. In N. Rumsey \& D. Harcourt, The Oxford Handbook of The Psychology of Appearance. Oxford University Press.

UNICEF (Ed.). (2011). Adolescence: An age of opportunity. UNICEF.

van Dalen, M., Dierckx, B., Pasmans, S. G. M. A., Aendekerk, E. W. C., Mathijssen, I. M. J., Koudstaal, M. J., Timman, R., Williamson, H., Hillegers, M. H. J., Utens, E. M. W. J., \& Okkerse, J. M. E. (2020). Anxiety and depression in adolescents with a visible difference: A systematic review and meta-analysis. Body Image, 33, 38-46. https://doi.org/10.1016/j.bodyim.2020.02.006

Veiga, F. H., \& Leite, A. (2016). Escala de avaliação do autoconceito de adolescentes: Versão do Piers-Harris reduzida a 30 itens em escala 1-6. In F. Veiga (Ed.), Envolvimento dos Alunos na Escola: Perspetivas da Psicologia e Educação-Motivação para o Desempenho Académico (pp. 77-89). Instituto de Educação da Universidade de Lisboa.

Wehrle, K., \& Fasbender, U. (2019). Self-Concept. In V. Zeigler-Hill \& T. K. Shackelford (Eds.), Encyclopedia of personality and individual differences (pp. 1-5). Springer International Publishing. https://doi.org/10.1007/978-3-319-28099-8_2001-1

Wong, D. L., Hockenberry, M. J., \& Wilson, D. (Eds.). (2011). Wong's nursing care of infants and children (9th ed). Mosby/Elsevier. 